\title{
Rationing on the Fly: The Opportunity Cost of Clinical Guidelines
}

\author{
Theodore G. Ganiats, MD, and Jennifer A. Kempster, MPhil
}

\section{Time as a Limited Resource}

Clinical guidelines translate research findings and expert opinion into recommendations for management of a specific disease, disease group, or health risk. There are thousands of guidelines in the $\mathrm{Na}$ tional Guideline Clearinghouse, and updated and new ones are constantly added. In an ideal world all indicated guidelines would be followed for every patient, but implementing a new guideline often requires the expenditure of care and resources, including the use of time by both the patient and provider. From the patient perspective, the question remains: How many appropriate guidelines can any one patient follow? This is especially true for those with comorbidities and multiple indicated guidelines. ${ }^{1}$ From the provider perspective, similar questions arise: How many appropriate guidelines can be followed in one patient visit? Which are most beneficial? ${ }^{1,2}$ A growing number of guidelines and a fixed amount of time means that at some point not all guidelines can be implemented.

The decision of what to discontinue to be able to add a guideline is a difficult task. While some guidelines offer suggestions for managing a patient in the face of comorbidity (eg, hypertension in those with diabetes), guidelines do not help providers prioritize therapeutic options in patients with multiple diseases (eg, how do you prioritize heart failure recommendations in a patient with diabetes and chronic obstructive pulmonary disease?). In addition, guideline evaluations assess only the guideline's effects on disease-specific outcomes not

This article was externally peer reviewed.

Submitted 14 October 2012; revised 8 January 2014; accepted 31 March 2014.

From the Department of Family and Preventive Medicine, University of California-San Diego, La Jolla.

Funding: none.

Conflict of interest: none declared.

Corresponding author: Theodore G. Ganiats, MD, Department of Family and Preventive Medicine, University of California-San Diego, 9500 Gilman Drive, La Jolla, CA 92093-0622 (E-mail: tganiats@ucsd.edu). its effect on the whole patient. This means the comparative effect on individual patient outcomes is unknown. Ensuring the care provided maximizes health benefits for each patient requires an accurate assessment by the provider of the value of the discontinued guideline. This entails forecasting individual patient outcomes across all health domains for every possible combination of guidelines, including those administered by other providers. Such an evaluation is clearly not possible for a provider to do on a patient-by-patient, appointment-by-appointment basis, but it is what the current guideline framework calls for.

There are potential ways to increase the supply of provider time to meet the additional demand for care imposed by a new guideline. These include lengthening the workday, improving efficiency (indeed, many guidelines help improve efficiency), and adding providers. However, there are natural limits to all these options; providers cannot work around the clock, efficiency has a natural limit (and guidelines are not usually designed with efficiency in mind), and underuse of the trained provider workforce is very low. ${ }^{3}$ So when providers are faced with new guidelines, they must decide what care to give in the time available. This requires removing other health care, which means the addition of a new guideline results in some care not being provided; care will be rationed. The discontinued care is the opportunity cost of implementing the new intervention.

\section{Time as a Limited Resource by Individual Patient Visit}

When a patient presents to a provider, the provider initiates a dialog that prioritizes the patient's issues. The provider then selects-often with the help of the patient-which issues will be discussed immediately and which will be postponed. For example, by prioritizing a discussion of the importance of folate supplementation with a woman contemplat- 
ing pregnancy, the provider may decide not to discuss the health hazards of her being overweight. In this situation, the provider tries to balance the potential benefits of one guideline with the lost or delayed benefits of another guideline for that specific patient. This is a difficult task under ideal circumstances, let alone during a visit in the middle of a busy day. Basically, what to do (the guideline) may be based on evidence, but we delete guidelines in an ad hoc manner. Postponing the unimplemented or discontinued guideline may seem like a possible solution, but this may not be viable since it could result in an opportunity cost at a later visit. If the provider decides that both interventions must be implemented during the visit, the extra time will have to be subtracted from another patient or other patients later in the day.

\section{Time as a Limited Resource by Individual Provider}

Given that the length of a provider's workday is relatively fixed, the number of patient visits and the case mix influences the care received by all patients seen that day. On days where a provider sees fewer patients because of scheduling or when the case mix includes many patients with simple problems, the provider has more time available for each patient and can use this time to add new guidelines to patient care. On days when there are extra patients or appointments are, on average, running long, the provider tends to spend less time with some patients, essentially rationing their care. Although this rationing is often second nature to experienced clinicians who confidently prioritize care at the individual patient level, it is unknown whether this prioritization maximizes benefits for individual patients.

\section{Problems with Evaluating the Effect of a Guideline}

As previously discussed, there is a tendency to evaluate a guideline's effects, both benefits and harms, in the context of a single disease or behavior. One might implement a program that is effective from this narrow perspective but, from a broader view, actually reduces the overall benefits to some patients. In other words, the evaluation usually informs a narrow question (eg, "Did the rate of diabetic retinopathy decrease?"), not the effect on the patient (eg, "Is the patient better off overall?) or the total system (eg, "What is the effect on the health of my patient population?"). These latter questions are more relevant if the ultimate goal is to maxi- mize overall health as opposed to treating a single disease.

\section{Where Now?}

The benefits of guidelines are undisputed, and there is no question that they should be an essential element of health care planning. ${ }^{4}$ However, in the United States guidelines are usually developed without considering that a key limiting resource in health care provision is provider time and that an assessment of the comparative effectiveness of the alternative scenarios under consideration is needed.

That provider time is limited means that some guidelines are essentially unfunded (in terms of time) mandates, asking the provider to do more without giving additional time to do it. This unfunded mandate means (1) providers ration care provided to the same patient or other patients later in the day; (2) this rationing may be suboptimal since the effectiveness of the new guideline ignores the effect of what care will be dropped; (3) since providers do not record what was dropped to make room for the new service, one can never know the opportunity cost in terms of health, costs, or costeffectiveness of an intervention; and (4) the effect of performance measures, which are based on guidelines, also is unknown.

The solutions are not obvious. One approach is to evaluate guidelines from a broader perspective. However, this is impractical because there are so many potential scenarios and the methods to perform this evaluation do not exist. Clearly, more research on how busy clinicians prioritize and make choices about the implementation of clinical guidelines with limited information, and how this information processing can be improved, is needed. While electronic medical records and computer system prompts have been developed to alert providers to the guidelines indicated for each patient, ${ }^{5}$ these methods do not prioritize interventions to maximize effectiveness for each patient or across a population. This is not a computer systems problem; it is a lack of data on the relative effectiveness of individual guidelines. Similarly, the National Guidelines Clearinghouse website includes a "compare guidelines" functionality. ${ }^{6}$ However, it is only possible to compare guidelines within the same subtopic, and the tool does not include a comparison of effectiveness. Data might be available from practice-based research networks, but to date such data (and such functionality of the networks) are still lacking. 
Clearly, a more realistic evaluation of the total effect of guidelines on individuals and across populations will require the development of new methods. Until this is done, the true impact of clinical guidelines cannot be known.

\section{References}

1. Yarnall KSH, Pollak KI, Ostbye T, Krause KM, Michener JL. Primary care: is there enough time for prevention? Am J Public Health 2003;95(4):635-41.

2. Cabana MD, Rand CS, Powe NR, Wu AW, Wilson MH, Abboud PC, Rubin HR. Why don't physicians follow clinical practice guidelines? A framework for improvement. JAMA 1998;282:1458-65.

3. Unemployment rates, a detailed look. The Wall Street Journal, 2010. Available from: http://online. wsj.eom/article/SB10001424052748703791904576 075652301620440.html. Accessed August 15, 2012.

4. Committee on Standards for Developing Trustworthy Clinical Practice Guidelines, Institute of Medicine. Summary. Clinical practice guidelines we can trust. Washington, DC: National Academies Press, 2011.

5. Damiani G, Pinnarelli L, Colosimo SC, et al. The effectiveness of computerized clinical guidelines in the process of care: a systematic review. BMC Health Serv Res 2010;10:2.

6. Agency for Healthcare Research and Quality. National Guideline Clearinghouse. Compare guidelines. Available at: http://www.guidelines.gov/compare/index.aspx. Accessed August 15, 2012.

doi: 10.3122/jabfm.2014.04.120282

\section{Counterpoint: Rationing on the Fly: The Opportunity Cost of Clinical Guidelines}

\section{Larry Culpepper, $M D, M P H$}

Adoption of new guidelines must be balanced by the reduction of other care during visits since such care delivery is a zero sum game bounded by time. The resulting deletion of care is done ad hoc in the midst of busy visits. This results in a problemrationing - the solution for which is not obvious, but at least now we have labeled the problem.

From the Department of Family Medicine, Boston University Medical Center, Boston, MA.

Funding: none.

Conflict of interest: none declared.

Corresponding author: Larry Culpepper, MD, MPH, Department of Family Medicine, Boston University Medical Center, Dowling 5, One Boston Medical Center Place, Boston, MA 02118-2393 (E-mail: laculpep@bu.edu).
These are the basic tenets of the Commentary by Ganiats and Kempster. ${ }^{1}$ However, it is unclear that these tenets are valid or adequate to define the problem or guide the evolution of practice.

Several considerations are relevant. First, the care we provide patients is driven by the number of problems they have, not by the number of guidelines available. Second, the number of problems we address during a single visit (or during any other interval of time) often is determined by the patient's capacity to engage in the care required, including at the behavioral level. Third, our selection of problems to tackle is a complex process involving clinician-guided patient prioritizing and decision making, again bounded by the capacity of the patient as well as the clinician.

Ganiats and Kempster's dilemma of the increasing number of guidelines applicable to the patient with multiple problems is inherently an issue related to the complexity of such patients rather than to the guidelines that might inform their care. It remains for clinicians to determine whether new guidelines are applicable or whether continuing previous "expert opinion" care or no care is preferable. New guidelines might make relevant evidence more accessible to clinicians. However, as Katerndahl ${ }^{2}$ notes, the complexity of such patients requires primary care clinicians to have the ability to provide high-value care integrated across problems rather than simply adding to the checklist another guideline for a "complicated" patient. The number of problems, not the number of guidelines, is the issue.

Ganiats and Kempster's tenets might be applicable particularly if clinicians and patients implement new guidelines for problems that were not under care. To the degree that problems were being addressed clinically, guidelines might improve care and save time. Guideline-driven improvements to care, while possibly requiring increased time initially, is likely to save time in the long term to the degree that worsening morbidity is prevented. Similarly, initiating care for problems previously not under care will take time early but might save time later. Consequently, the potential detrimental impact derived from Ganiats and Kempster's concern regarding compensatory timedriven abandonment of other care arises primarily as a trade-off between time consumed now to improve health with time savings later. The key variable is the time horizon for summing the trade-off 